\title{
Casimir energy and dilute dielectric ball
}

\author{
Valery N. Marachevsky* \\ Department of Theoretical Physics \\ St.Petersburg State University \\ 198904 St.Petersburg, Russia
}

\begin{abstract}
General formalism of quantum field theory and addition theorem for Bessel functions are applied to derive formula for CasimirPolder energy of interaction between a polarizable particle and a dilute dielectric ball. The equivalence of dipole-dipole interaction and Casimir energy for dilute homogeneous dielectrics is shown. A novel method is used to derive Casimir energy of a dilute dielectric ball without divergences in calculations. Physically realistic model of a dilute ball is discussed. Different approaches to the calculation of Casimir energy of a dielectric ball are reviewed.
\end{abstract}

PACS numbers: 03.70.+k, 11.10.Gh, 12.20.-m, 12.20.Ds, 42.50.Lc

*E-mail: root@VM1485.spb.edu 


\section{Introduction}

The study of spherical geometry in Casimir effect meets a lot of technical problems, the most difficult one is the problem of divergences, which appear in many expressions. The special interest to the subject raised after the series of articles by Julian Schwinger where he had suggested a connection between Casimir effect and sonoluminescence [1, 2]. Different regularizations were used to obtain finite results in spherical geometry, however, results of the calculations strongly depended on the regularization scheme and regu-

larization parameters (a discussion of divergences and their regularization can be found in [3]). In the pesent paper we show that it is necessary that regularization parameters be present even in final expressions, they have real physical meaning and we will clarify it.

We study a dielectric nonmagnetic ball of radius $a$ and permittivity $\varepsilon$, surrounded by a vacuum. The ball is dilute, i.e. all final expressions are obtained under the assumption $\varepsilon-1 \ll 1$. Dispersion is essential in the following, so the permittivity $\varepsilon$ depends on frequency.

In section 2 we follow the formalism which was developed by E.Lifshitz et.al. [4] and K.Milton et.al.[5]. We start from a short overview of known facts. Then we derive Casimir-Polder type energy of interaction between a dielectric ball and a particle of constant polarizability $\alpha$, which is placed at the distance $r>a$ from the centre of the ball. The addition theorem for Bessel functions is used. Another application of this technique can be found in [6] where Casimir energy of polarizable particle located in the neighbourhood of perfectly conducting wedge was calculated. In section 3 the equivalence of dipole-dipole interaction and Casimir energy in the lowest order $(\varepsilon-1)^{2}$ is proved for homogeneous dielectric media. This fact is used in section 4 to calculate non-dispersive contribution to the energy, dispersive contribution is briefly discussed. We argue that Casimir surface force is attractive in a realistic model of dielectric ball and includes volume and surface terms in the order $(\varepsilon-1)^{2}$.

Various regularizations are reviewed in the Appendix.

We put $\hbar=c=1$. Heaviside-Lorentz units are used. 


\section{Polarizable particle and a ball}

The change in the ground state energy $E$ of the system under the infinitesimal variation of $\varepsilon$ is

$$
\delta E=\frac{i}{2} \int d^{3} \mathbf{x} \int_{-\infty}^{+\infty} \frac{d \omega}{2 \pi} \delta \varepsilon(\mathbf{x}, \omega) D_{p p}(\varepsilon, \mathbf{r}, \mathbf{r}, \omega) .
$$

Here $D_{j k}(\varepsilon, \mathbf{r}, \mathbf{r}, \omega)$ is a Fourier component of electric field propagator trace, it satisfies equations

$$
\left[\varepsilon(\mathbf{r}, \omega) \omega^{2} \delta_{j m}-\operatorname{rot}_{j l} \operatorname{rot}_{l m}\right] D_{m k}\left(\varepsilon, \mathbf{r}, \mathbf{r}^{\prime}, \omega\right)=-\omega^{2} \delta\left(\mathbf{r}-\mathbf{r}^{\prime}\right) \delta_{j k}
$$

The system of equations for this Green's function was discussed extensively

in [4, 5] (there is a discussion of these equations in the next section). The solution of this system for spherical geometry with standard boundary conditions classically imposed at $r=a$ can be written as in [7] ( $\delta$-functions are omitted since we are interested in the limit $\mathbf{r} \rightarrow \mathbf{r}^{\prime}$ ):

$$
\begin{aligned}
D_{j k}\left(\varepsilon, \mathbf{r}, \mathbf{r}^{\prime}, \omega\right)=-\sum_{l=1}^{\infty} \sum_{m=-l}^{l}\left(\omega^{2} F_{l}\left(r, r^{\prime}\right) X_{j l m}(\Omega) X_{k l m}^{*}\left(\Omega^{\prime}\right)+\right. \\
\left.+\frac{1}{\varepsilon} \operatorname{rot}_{\mathbf{r}} \operatorname{rot}_{\mathbf{r}^{\prime}} G_{l}\left(r, r^{\prime}\right) X_{j l m}(\Omega) X_{k l m}^{*}\left(\Omega^{\prime}\right)\right) .
\end{aligned}
$$

Here we have used the following notations $\left(X_{i l m}(\Omega)\right.$ are vector spherical harmonics; $j_{l}(r), h_{l}^{(1)}(r)$ are spherical Bessel functions, $\tilde{e}_{l}(r)=r h_{l}^{(1)}(r), \tilde{s}_{l}(r)=$ $r j_{l}(r)$ are Riccati-Bessel functions [8]):

$$
\begin{gathered}
X_{i l m}(\Omega)=\frac{1}{\sqrt{l(l+1)}}\left(\mathbf{L} Y_{l m}(\Omega)\right)_{i} \\
F_{l}, G_{l}=\left\{\begin{array}{l}
i k j_{l}\left(k r_{<}\right)\left[h_{l}^{(1)}\left(k r_{>}\right)-A_{F, G} j_{l}\left(k r_{>}\right)\right], k=|\omega| \sqrt{\varepsilon}, \quad r, r^{\prime}<a, \\
i|\omega| h_{l}^{(1)}\left(|\omega| r_{>}\right)\left[j_{l}\left(|\omega| r_{<}\right)-B_{F, G} h_{l}^{(1)}\left(|\omega| r_{<}\right)\right], \quad r, r^{\prime}>a,
\end{array}\right. \\
A_{F}=\frac{\tilde{e}_{l}(|\omega| \sqrt{\varepsilon} a) \tilde{e}_{l}^{\prime}(|\omega| a)-\sqrt{\varepsilon} \tilde{e}_{l}(|\omega| a) \tilde{e}_{l}^{\prime}(|\omega| \sqrt{\varepsilon} a)}{\Delta_{l}}, \\
B_{F}=\frac{\tilde{s}_{l}(|\omega| \sqrt{\varepsilon} a) \tilde{s}_{l}^{\prime}(|\omega| a)-\sqrt{\varepsilon} \tilde{s}_{l}(|\omega| a) \tilde{s}_{l}^{\prime}(|\omega| \sqrt{\varepsilon} a)}{\Delta_{l}}, \\
A_{G}=\frac{\sqrt{\varepsilon} \tilde{e}_{l}(|\omega| \sqrt{\varepsilon} a) \tilde{e}_{l}^{\prime}(|\omega| a)-\tilde{e}_{l}(|\omega| a) \tilde{e}_{l}^{\prime}(|\omega| \sqrt{\varepsilon} a)}{\tilde{\Delta}_{l}} \\
B_{G}=\frac{\sqrt{\varepsilon} \tilde{s}_{l}(|\omega| \sqrt{\varepsilon} a) \tilde{s}_{l}^{\prime}(|\omega| a)-\tilde{s}_{l}(|\omega| a) \tilde{s}_{l}^{\prime}(|\omega| \sqrt{\varepsilon} a)}{\tilde{\Delta}_{l}}
\end{gathered}
$$




$$
\begin{aligned}
& \Delta_{l}=\tilde{s}_{l}(|\omega| \sqrt{\varepsilon} a) \tilde{e}_{l}^{\prime}(|\omega| a)-\sqrt{\varepsilon} \tilde{s}_{l}^{\prime}(|\omega| \sqrt{\varepsilon} a) \tilde{e}_{l}(|\omega| a), \\
& \tilde{\Delta}_{l}=\sqrt{\varepsilon} \tilde{s}_{l}(|\omega| \sqrt{\varepsilon} a) \tilde{e}_{l}^{\prime}(|\omega| a)-\tilde{s}_{l}^{\prime}(|\omega| \sqrt{\varepsilon} a) \tilde{e}_{l}(|\omega| a),
\end{aligned}
$$

differentiation is taken over the whole argument.

When the point particle of constant polarizability $\alpha$ is inserted into the point $\mathbf{r},|\mathbf{r}|>a$ from the centre of the ball, the energy change is given by (四) with $\delta \epsilon=4 \pi \alpha \delta^{3}(\mathbf{r}-\mathbf{x})$. However, the contact terms (empty space contribution) have to be subtracted from (11), i.e. when physical quantities are calculated in the region $r, r^{\prime}>a$ the term $i|\omega| h_{l}^{(1)}\left(|\omega| r_{>}\right) j_{l}\left(|\omega| r_{<}\right)$has to be subtracted from (5) (for $r, r^{\prime}<a$ in full analogy the term $i k j_{l}\left(k r_{<}\right) h_{l}^{(1)}\left(k r_{>}\right)$ should be subtracted from (5)). Doing so, we have to substitute $\tilde{F}_{l}, \tilde{G}_{l}$ instead of $F_{l}, G_{l}$ in all expressions, where

$$
\tilde{F}_{l}, \tilde{G}_{l}= \begin{cases}-i A_{F, G} k j_{l}\left(k r_{<}\right) j_{l}\left(k r_{>}\right), k=|\omega| \sqrt{\varepsilon}, & r, r^{\prime}<a \\ -i B_{F, G}|\omega| h_{l}^{(1)}\left(|\omega| r_{>}\right) h_{l}^{(1)}\left(|\omega| r_{<}\right), & r, r^{\prime}>a .\end{cases}
$$

The Casimir-Polder energy of this configuration is

$$
\begin{aligned}
& E_{1}(r, a)=i \alpha \int_{-\infty}^{+\infty} d \omega D_{p p}(\varepsilon, \mathbf{r}, \mathbf{r}, \omega)=\frac{\alpha}{i} \int_{-\infty}^{+\infty} d \omega \sum_{l=1}^{\infty} \frac{2 l+1}{4 \pi} \times \\
& \quad \times\left.\left(\omega^{2} \tilde{F}_{l}\left(r, r^{\prime}\right)+l(l+1) \frac{\tilde{G}_{l}\left(r, r^{\prime}\right)}{r r^{\prime}}+\frac{1}{r r^{\prime}} \frac{\partial}{\partial r} r \frac{\partial}{\partial r^{\prime}}\left(r^{\prime} \tilde{G}_{l}\left(r, r^{\prime}\right)\right)\right)\right|_{r^{\prime} \rightarrow r} .
\end{aligned}
$$

We perform a Euclidean rotation then: $\omega \rightarrow i \omega$,

$$
\tilde{s}_{l}(x) \rightarrow s_{l}(x)=\sqrt{\frac{\pi x}{2}} I_{l+1 / 2}(x), \tilde{e}_{l}(x) \rightarrow e_{l}(x)=\sqrt{\frac{2 x}{\pi}} K_{l+1 / 2}(x) .
$$

Let $x=\omega a$. For $E_{1}(r, a)$ we obtain

$$
\begin{array}{rl}
E_{1}(r, a)=\frac{2 \alpha}{a} \int_{0}^{+\infty} d & x \sum_{l=1}^{+\infty} \frac{2 l+1}{4 \pi}\left[\frac{x}{a r^{2}} e_{l}^{2}(x r / a) B_{F}-\right. \\
& \left.-\frac{l(l+1) a}{r^{4} x} e_{l}^{2}(x r / a) B_{G}-\frac{x}{r^{2} a}\left(e_{l}^{\prime}(x r / a)\right)^{2} B_{G}\right] .
\end{array}
$$

This expression can be transformed to a simple formula in the limit $\varepsilon-1 \ll 1$. The functions $B_{F}$ and $B_{G}$ are proportional to $(\varepsilon-1)$ in this limit. To proceed, the following addition theorem for Bessel functions [9] is useful:

$$
\begin{gathered}
u(p, k, x, \rho) \equiv \sum_{l=0}^{+\infty}(2 l+1) s_{l}(x p) e_{l}(x k) P_{l}(\cos \theta)=\frac{x e^{-x \rho} p k}{\rho} \\
\rho=\sqrt{p^{2}+k^{2}-2 p k \cos \theta}
\end{gathered}
$$


To our knowledge this formula was first used in Casimir effect calculations in [10, where it was applied to analytic calculation of Casimir energy of perfectly conducting spherical shell and dilute dielectric ball satisfying $\varepsilon \mu=1$.

In our case it can be applied as follows. The simple identity holds $(k>$ $p>0$ for definiteness):

$$
\begin{aligned}
\int d x \sum_{l=0}^{+\infty}(2 l+1) f(x) & s_{l}(x p) e_{l}(x k) s_{l}(x p) e_{l}(x k)= \\
= & \frac{1}{2} \int_{k-p}^{k+p} \frac{d \rho \rho}{p k} \int d x f(x) u(p, k, x, \rho) u(p, k, x, \rho)
\end{aligned}
$$

where we have used

$$
\begin{gathered}
\int_{-1}^{1} d(\cos \theta) P_{l}(\cos \theta) P_{m}(\cos \theta)=\frac{2}{2 l+1} \delta_{l m}, \\
\int_{-1}^{+1} d(\cos \theta) \cdots=\int_{k-p}^{k+p} \frac{d \rho \rho}{p k} \cdots .
\end{gathered}
$$

Only the first order $\sim(\varepsilon-1)$ is needed in $E_{1}$. We put $k=r / a, p=1$ and use (18) and its obvious generalizations in (15) to calculate $E_{1}$. Finally we get

$$
E_{1}(r, a)=-\frac{23}{15} \alpha \frac{\varepsilon-1}{4 \pi} \frac{a^{3}\left(5 r^{2}+a^{2}\right)}{r(r+a)^{4}(r-a)^{4}}, r>a .
$$

Substitution $\varepsilon-1=4 \pi N_{\text {mol }} \alpha_{\text {ball }}$ in the limit $r \gg a$ yields

$$
\left.E_{1}(r, a)\right|_{r \gg a}=N_{m o l}\left(\frac{4 \pi a^{3}}{3}\right) \frac{-23 \alpha \alpha_{\text {ball }}}{4 \pi r^{7}}=N_{m o l}\left(\frac{4 \pi a^{3}}{3}\right) E_{C a s-P o l} .
$$

Thus in this limit the famous Casimir-Polder energy of interaction between two polarizable particles $E_{C a s-P o l}$ [11] can be obtained directly from (22).

Certainly, this is not simply a coincidence, and next section is devoted to the proof of equivalence of dipole-dipole interaction and Casimir energy in the order $(\varepsilon-1)^{2}$.

\section{Dipole interaction and Casimir energy}

We define differential operator

$$
L_{j m}(\varepsilon, \mathbf{r}, i \omega)=\left[\varepsilon(\mathbf{r}, i|\omega|) \omega^{2} \delta_{j m}+\operatorname{rot}_{j l} \operatorname{rot}_{l m}\right] .
$$




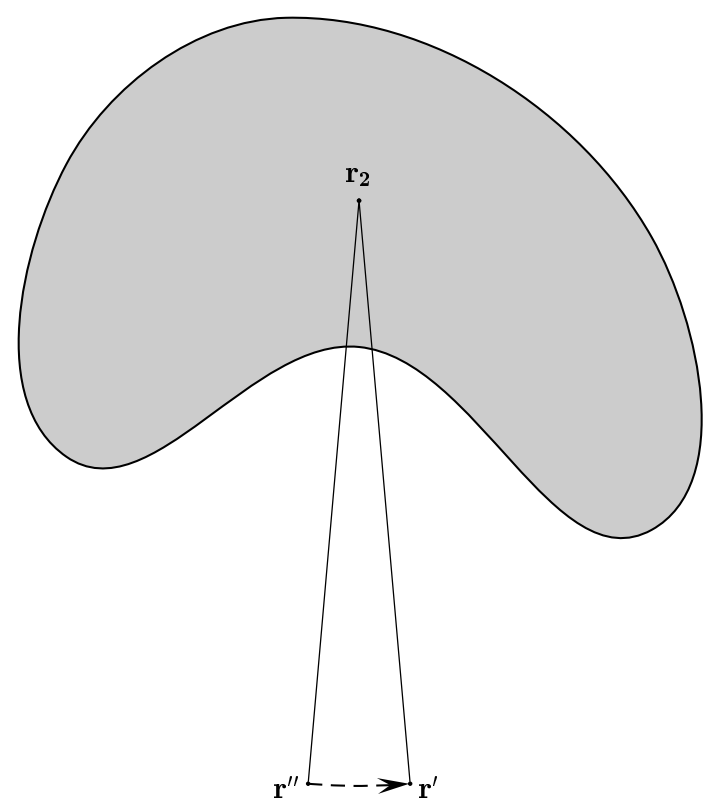

Figure 1: dielectric area is shaded; point particle is located at point $\mathbf{r}^{\prime}$

Equations for electric field propagator are well known due to works [4, [5] :

$$
L_{j m}(\varepsilon, \mathbf{r}, i \omega) D_{m k}\left(\varepsilon, \mathbf{r}, \mathbf{r}^{\prime}, i \omega\right)=-\omega^{2} \delta\left(\mathbf{r}-\mathbf{r}^{\prime}\right) \delta_{j k} .
$$

Suppose that $\varepsilon$ has changed in the point $\mathbf{r}_{2}$. The changed system satisfies equation

$$
\begin{array}{r}
L_{j m}\left(\varepsilon+\delta \varepsilon(i|\omega|) \delta\left(\mathbf{r}_{2}-\mathbf{r}\right), \mathbf{r}, i \omega\right) \tilde{D}_{m k}\left(\varepsilon+\delta \varepsilon(i|\omega|) \delta\left(\mathbf{r}_{2}-\mathbf{r}\right), \mathbf{r}, \mathbf{r}^{\prime}, i \omega\right)= \\
=-\omega^{2} \delta\left(\mathbf{r}-\mathbf{r}^{\prime}\right) \delta_{j k}
\end{array}
$$

Let us define $\delta D_{j k}\left(\mathbf{r}, \mathbf{r}^{\prime}, i \omega\right)$ as a term proportional to $\delta \varepsilon(i|\omega|)$ in the expansion

$$
\widetilde{D}_{j k}=D_{j k}+\delta D_{j k}\left(\mathbf{r}, \mathbf{r}^{\prime}, i \omega\right)+O\left((\delta \varepsilon(i|\omega|))^{2}\right)
$$

If we consider equation (25) and write series in powers of constant $\delta \varepsilon(i|\omega|)$, in the first order we get the equality:

$$
\delta \varepsilon(i|\omega|) \delta\left(\mathbf{r}_{2}-\mathbf{r}\right) \omega^{2} D_{j k}\left(\varepsilon, \mathbf{r}, \mathbf{r}^{\prime}, i \omega\right)+L_{j m}(\varepsilon, \mathbf{r}, i \omega) \delta D_{m k}\left(\mathbf{r}, \mathbf{r}^{\prime}, i \omega\right)=0 .
$$

We multiply equation (27) by $D_{p j}\left(\mathbf{r}^{\prime \prime}, \mathbf{r}, i \omega\right)$ on the left, integrate over $\mathbf{r}$ and sum over $j$. Using properties $D_{p j}\left(\mathbf{r}^{\prime \prime}, \mathbf{r}, i \omega\right)=D_{j p}\left(\mathbf{r}, \mathbf{r}^{\prime \prime}, i \omega\right), L_{j m}^{\dagger}=L_{m j}$ and equation (24) it is straightforward to obtain

$$
\delta D_{p k}\left(\mathbf{r}^{\prime \prime}, \mathbf{r}^{\prime}, i \omega\right)=D_{p j}\left(\varepsilon, \mathbf{r}^{\prime \prime}, \mathbf{r}_{2}, i \omega\right) \delta \varepsilon(i|\omega|) D_{j k}\left(\varepsilon, \mathbf{r}_{2}, \mathbf{r}^{\prime}, i \omega\right) .
$$


Now suppose that $D_{m k}$ satisfies (24) with $\varepsilon=1$ in all space. Then we insert dielectric of arbitrary shape with constant dielectric permittivity $\varepsilon(i|\omega|)$ so that $\delta \varepsilon(i|\omega|)=\varepsilon(i|\omega|)-1$. For this case $\delta$-function in (27) has to be substituted by function equal to 0 outside the dielectric and equal to 1 inside the dielectric. Denoting $D_{p k}^{(0)}\left(\mathbf{r}^{\prime \prime}, \mathbf{r}^{\prime}, i \omega\right) \equiv D_{p k}\left(\varepsilon=1, \mathbf{r}^{\prime \prime}, \mathbf{r}^{\prime}, i \omega\right)$, we get the formula

$$
\begin{aligned}
& D_{p k}\left(\varepsilon, \mathbf{r}^{\prime \prime}, \mathbf{r}^{\prime}, i \omega\right)-D_{p k}^{(0)}\left(\mathbf{r}^{\prime \prime}, \mathbf{r}^{\prime}, i \omega\right)= \\
& =\int d^{3} \mathbf{r}_{2} D_{p j}^{(0)}\left(\mathbf{r}^{\prime \prime}, \mathbf{r}_{2}, i \omega\right)(\varepsilon(i|\omega|)-1) D_{j k}^{(0)}\left(\mathbf{r}_{2}, \mathbf{r}^{\prime}, i \omega\right)+O\left((\varepsilon(i|\omega|)-1)^{2}\right)
\end{aligned}
$$

Figure 1 illustrates formula (29). When $\mathbf{r}^{\prime \prime} \Rightarrow \mathbf{r}^{\prime}$ the formula for Casimir energy of interaction between a polarizable particle located at point $\mathbf{r}^{\prime}$ and dielectric can be written as

$$
\begin{aligned}
E & =-\left.\int_{-\infty}^{+\infty} d \omega \alpha_{1}\left(\mathbf{r}^{\prime}, i|\omega|\right)\left(D_{p p}\left(\varepsilon, \mathbf{r}^{\prime \prime}, \mathbf{r}^{\prime}, i \omega\right)-D_{p p}^{(0)}\left(\mathbf{r}^{\prime \prime}, \mathbf{r}^{\prime}, i \omega\right)\right)\right|_{\mathbf{r}^{\prime \prime} \rightarrow \mathbf{r}^{\prime}}= \\
& =\int d^{3} \mathbf{r}_{2} \rho\left(\mathbf{r}_{2}\right) U\left(\mathbf{r}^{\prime}, \mathbf{r}_{2}\right)+O\left(\alpha^{3}\right)
\end{aligned}
$$

where

$$
U\left(\mathbf{r}^{\prime}, \mathbf{r}_{2}\right)=-\int_{-\infty}^{+\infty} d \omega 4 \pi \alpha_{1}\left(\mathbf{r}^{\prime}, i|\omega|\right) \alpha_{2}\left(\mathbf{r}_{2}, i|\omega|\right)\left(D_{p j}^{(0)}\left(\mathbf{r}^{\prime}, \mathbf{r}_{2}, i \omega\right)\right)^{2} .
$$

Equation for potential (31) can also be obtained from dipole-dipole interaction of two neutral atoms [12]. We refer the reader to this book. If we denote $r=\left|\mathbf{r}_{2}-\mathbf{r}^{\prime}\right|$, then (see [12])

$U(r)=-\frac{1}{\pi r^{2}} \int_{0}^{+\infty} \omega^{4} \alpha_{1}(i \omega) \alpha_{2}(i \omega) e^{-2 \omega r}\left[1+\frac{2}{\omega r}+\frac{5}{(\omega r)^{2}}+\frac{6}{(\omega r)^{3}}+\frac{3}{(\omega r)^{4}}\right] d \omega$

If we define atomic absorption frequency as $\omega_{0}$, then $\lambda \sim 1 / \omega_{0}$ is a characteristic absorption wavelength in the spectrum of interacting atoms. For distances $r \gg \lambda$ the main contribution to (32) gives the frequency region $\omega \lesssim 1 / r \ll 1 / \lambda$. In this frequency region according to general properties of dielectric permittivity on imaginary axis it is poissible to use $\alpha_{1}(0)$ and $\alpha_{2}(0)$ instead of $\alpha_{1}(i \omega)$ and $\alpha_{2}(i \omega)$, so (32) for $r \gg \lambda$ yields Casimir-Polder potential

$$
\left.U(r)\right|_{r \gg \lambda}=-\frac{23}{4 \pi} \frac{\alpha_{1}(0) \alpha_{2}(0)}{r^{7}}
$$


For $\lambda_{a t \text {. }} \ll r \ll \lambda\left(\lambda_{a t}\right.$. is atomic size $)$ frequencies $\omega \sim 1 / \lambda$ give essential

contribution to (32). Then the last term in (32) gives main contribution and

$$
\left.U(r)\right|_{r \ll \lambda}=-\frac{3}{\pi r^{6}} \int_{0}^{+\infty} d \omega \alpha_{1}(i \omega) \alpha_{2}(i \omega)
$$

Formulas (33) and (34) are non-dispersive and dispersive contributions to the energy of interaction of two neutral atoms.

\section{Energy of a dilute ball}

The change in the ground state energy of a dilute dielectric ball is generally equal

$$
d E=\left.\frac{d E}{d V}\right|_{\varepsilon=\text { const }} d V+\left.\frac{d E}{d \varepsilon}\right|_{V=\text { const }} d \varepsilon,
$$

where $V$ is the volume of the ball. Usually only the first term had been taken into account in the calculations of Casimir force and, as a consequence, the number of particles inside the ball wasn't conserved and the repulsive force resulted in most calculations. However, from physical considerations all atoms constituting the ball interact at large distances by attractive Casimir-Polder potential, so non-dispersive component of the force should be attractive.

Conservation of the number of atoms inside the ball is equivalent to the conditions

$$
(\varepsilon-1) V=\text { const }, \quad d \varepsilon=-\frac{(\varepsilon-1) d V}{V}
$$

We start from the first term contribution to (35) which we denote $d E_{1}$. Figure 2 clarifies designations. When particles are inserted into the (infinitely small) shaded area near point $B$, they interact via Casimir-Polder (retarded) potential with particles inside a ball of radius $a$ separated by distances $r \gtrsim \lambda$ from the point $B$, i.e. they interact via Casimir-Polder potential with particles inside the shaded region of the ball (regions $I+I I$ ). Particles separated from the point $B$ by distances $\lambda_{\text {at. }}<r_{\text {interatomic }} \ll r \lesssim \lambda$ interact with particles near point $B$ via van der Waals (non-retarded) potential. Obviously the situation is simplified for clarity here. Generally one has to take the concrete model of dispersion for dielectric permittivity, integrate interaction of particles near point $B$ via potential (31) with particles separated by distances greater than interatomic distances from point $B$ and calculate the energy change according to formula (35). As we are not intending to study specific model of dispersion here but rather want to get a suitable approximation for 


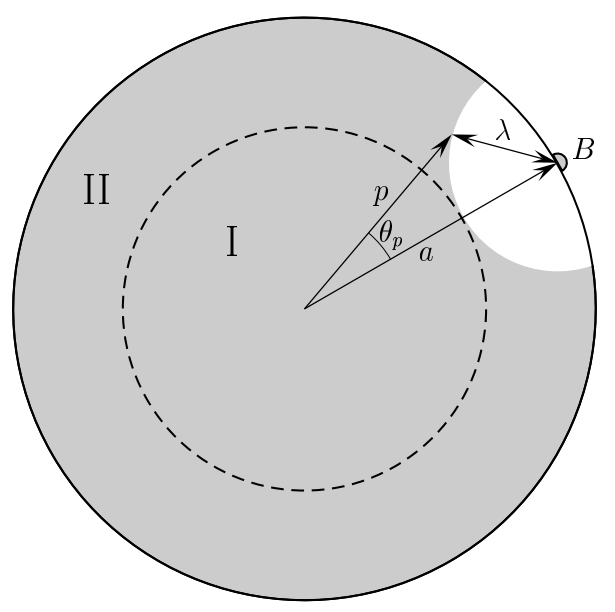

Figure 2: contribution to $d E_{1 \text { non-disp. }}$

low-frequency contribution to the energy, we approximate area of integration by its division on the retarded region $(r \gtrsim \lambda)$ and non-retarded region $\left(r_{\text {interatomic }} \lesssim r \lesssim \lambda\right)$. According to discussion in the previous section, for distances $r \gtrsim \lambda$ the frequency dispersion can be neglected, so this region in our approximation gives non-dispersive contribution to the energy, the region $r_{\text {interatomic }} \lesssim r \lesssim \lambda$ gives main dispersive contribution to the energy.

Area of integration for non-dispersive case is shaded on fig.2, so in our approximation the non-dispersive contribution to $d E_{1}$ can be written as

$$
\begin{aligned}
& d E_{1 \text { non-disp. }}=4 \pi a^{2} d a \int_{I+I I} d^{3} \mathbf{r} \frac{-23 \alpha\left(\mathbf{r}_{B}, \omega=0\right) \rho\left(\mathbf{r}_{B}\right) \alpha(\mathbf{r}, \omega=0) \rho(\mathbf{r})}{4 \pi\left|\mathbf{r}_{B}-\mathbf{r}\right|^{7}}= \\
&=4 \pi a^{2} d a \frac{(\varepsilon(\omega=0)-1)^{2}}{(4 \pi)^{2}} \int_{I+I I} d^{3} \mathbf{r} \frac{-23}{4 \pi\left|\mathbf{r}_{B}-\mathbf{r}\right|^{7}},
\end{aligned}
$$

where $\rho\left(\mathbf{r}_{B}\right)$ and $\rho(\mathbf{r})$ are densities of particles in a unit volume. The integral over the region $I$ can be simply obtained from (21) and arguments of the previous section. We just substitute $a \Rightarrow a-\lambda, r \Rightarrow a$ in formula (21) according to fig.2 and obtain:

$$
\int_{I} d^{3} \mathbf{r} \frac{-23}{4 \pi\left|\mathbf{r}_{B}-\mathbf{r}\right|^{7}}=-\frac{23}{15} \frac{(a-\lambda)^{3}\left(5 a^{2}+(a-\lambda)^{2}\right)}{a(2 a-\lambda)^{4} \lambda^{4}}
$$



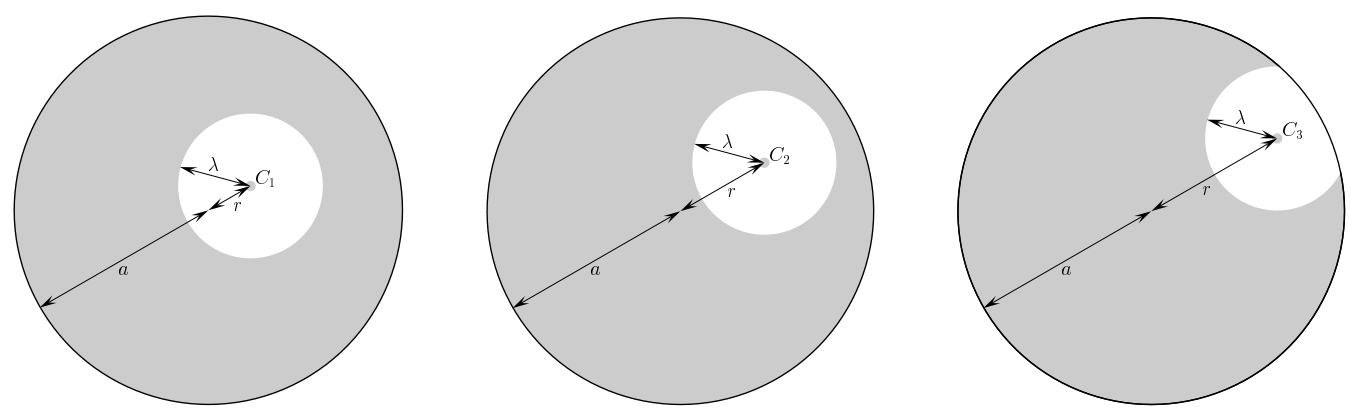

Figure 3: contributions to $d E_{2 \text { non-disp. }}$

The integral (37) over the region $I I$ can be calculated analytically using (20):

$$
\begin{aligned}
& \int_{I I} d^{3} \mathbf{r} \frac{1}{\left|\mathbf{r}_{B}-\mathbf{r}\right|^{7}}=\int_{a-\lambda}^{a} d p \int_{\theta_{p}}^{\pi} d \theta \frac{2 \pi p^{2} \sin \theta}{\left(a^{2}+p^{2}-2 a p \cos \theta\right)^{\frac{7}{2}}}= \\
& \quad=\int_{a-\lambda}^{a} d p \frac{2 \pi p}{a} \int_{\lambda}^{a+p} d \rho \frac{1}{\rho^{6}}=\frac{1}{480} \frac{\pi\left(5 \lambda^{4}+192 a^{4}-96 a^{3} \lambda\right)}{a^{4} \lambda^{4}}-\frac{1}{30} \frac{\pi(5 a-4 \lambda)}{a(2 a-\lambda)^{4}}
\end{aligned}
$$

From (37), (38) and (39) after integration over $a$ (keeping $\varepsilon=$ const) we obtain expression for $E_{1 \text { non-disp. }}$ :

$$
E_{1 \text { non-disp. }}=(\varepsilon-1)^{2}\left(-\frac{23}{128} \frac{V}{\pi^{2} \lambda^{4}}+\frac{23}{384} \frac{S}{\pi^{2} \lambda^{3}}+\frac{23}{1536} \frac{1}{\pi a}\right)+\text { const }
$$

Here $V$ and $S$ are volume and surface area of the ball respectively. Expression (40) looks similar to formula (49) obtained by G.Barton [13] in the framework of quantum mechanical perturbation theory. There is still a little difference between these formulas. At first sight it seems that in our approach it is impossible to find the term proportional to $1 / \lambda$ and this term has no influence on the force. However, this is not so. The term proportional to $1 / \lambda$ is determined uniquely in our approach when taking into account the second term in (35) and equation (36).

Now we turn to the second term in (35) and calculate its non-dispersive contribution to the energy change denoted by $d E_{2 \text { non-disp. }}$. Figure 3 illustrates different areas of integration over variable $r$ and analogous to fig. 2 in a sence - it is necessary to integrate Casimir-Polder potential between (infinitesimally small area around) point $C_{1}\left(C_{2}, C_{3}\right)$ and shaded area of the 
ball separated from point $C_{1}\left(C_{2}, C_{3}\right)$ by distances $r \gtrsim \lambda$ and then integrate over points $C_{1}, C_{2}, C_{3}$ inside the ball. This term can be written as follows:

$$
\left.d E_{2 \text { non-disp. }}\right|_{V=\text { const }}=\frac{\varepsilon-1}{4 \pi} \frac{\delta \varepsilon}{4 \pi} \iint_{\left|\mathbf{r}_{C}-\mathbf{r}\right| \geq \lambda} d V\left(\mathbf{r}_{C}\right) d V(\mathbf{r}) \frac{-23}{4 \pi\left|\mathbf{r}_{C}-\mathbf{r}\right|^{7}},
$$

where $\mathbf{r}_{C}$ and $\mathbf{r}$ are integrated over all points inside the ball of radius $a$ satisfying the condition $\left|\mathbf{r}_{C}-\mathbf{r}\right| \geq \lambda$.

To facilitate understanding of calculations in what follows we present major steps explicitly. Let us denote

$$
g(r, p, \rho)=4 \pi r^{2} \cdot 2 \pi p^{2} \frac{1}{r p \rho^{6}}
$$

and write $d E_{2 \text { non-disp. }}$ (after comparison with (39) and fig.3 everything should be clear):

$$
\begin{gathered}
d E_{2 \text { non-disp. }}=-\frac{23}{4 \pi} \frac{\varepsilon-1}{4 \pi} \frac{\delta \varepsilon}{4 \pi} \times \\
\times\left(\left(\int_{0}^{\lambda} d r \int_{\lambda-r}^{\lambda+r} d p \int_{\lambda}^{r+p} d \rho+\int_{0}^{\lambda} d r \int_{r+\lambda}^{a} d p \int_{p-r}^{r+p} d \rho\right) g(r, p, \rho) \quad \text { see fig. } 3.1 \quad+\right. \\
+\left(\int_{\lambda}^{a-\lambda} d r \int_{r+\lambda}^{a} d p \int_{p-r}^{p+r} d \rho+\int_{\lambda}^{a-\lambda} d r \int_{r-\lambda}^{r+\lambda} d p \int_{\lambda}^{r+p} d \rho+\right. \\
\left.+\int_{\lambda}^{a-\lambda} d r \int_{0}^{r-\lambda} d p \int_{r-p}^{r+p} d \rho\right) g(r, p, \rho) \quad \text { see fig.3.2 }+ \\
+\left(\int_{a-\lambda}^{a} d r \int_{r-\lambda}^{a} d p \int_{\lambda}^{r+p} d \rho+\int_{a-\lambda}^{a} d r \int_{0}^{r-\lambda} d p \int_{r-p}^{r+p} d \rho\right) g(r, p, \rho) \quad \text { see fig.3.3) }= \\
=2 \delta \varepsilon(\varepsilon-1)\left(-\frac{23}{128} \frac{V}{\pi^{2} \lambda^{4}}+\frac{23}{384} \frac{S}{\pi^{2} \lambda^{3}}-\frac{23}{384} \frac{1}{\pi \lambda}+\frac{23}{1536} \frac{1}{\pi a}\right) . \quad \text { (43) }
\end{gathered}
$$

The expression for non-dispersive force on a unit surface can be obtained using (35) and (36):

$$
\begin{aligned}
& F_{\text {non-disp. }}=-\frac{1}{4 \pi a^{2}} \frac{d E}{d a}=- \frac{1}{4 \pi a^{2}} \frac{d E_{1 \text { non-disp. }}+d E_{2 \text { non-disp. }}}{d a}= \\
&=-\frac{23}{128 \pi^{2} \lambda^{4}}\left(1-\frac{4}{3} \frac{\lambda}{a}+\frac{1}{2} \frac{\lambda^{3}}{a^{3}}-\frac{7}{48} \frac{\lambda^{4}}{a^{4}}\right) .
\end{aligned}
$$


This force is attractive for $\lambda \leq a$, which reflects attractive nature of CasimirPolder potential.

To calculate energy, one has to take into account equation (36) and integrate force. As a result,

$$
E_{n o n-d i s p .}=(\varepsilon-1)^{2}\left(-\frac{23}{128} \frac{V}{\pi^{2} \lambda^{4}}+\frac{23}{384} \frac{S}{\pi^{2} \lambda^{3}}-\frac{23}{384} \frac{1}{\pi \lambda}+\frac{23}{1536} \frac{1}{\pi a}\right) .
$$

The term proportional to $1 / \lambda$ is determined uniquely now. Moreover, comparison of (40) and (45) shows internal self-consistence of our approach.

\section{Conclusions}

The formulas for Casimir-Polder energy of interaction between a polarizable particle and a dilute dielectric ball (21), (22) and non-dispersive contribution to Casimir surface force on a dilute dielectric ball (44) are derived analytically. Our approach directly shows correspondence of this force and Casimir-Polder potential. In section 3 this topic has been investigated from general viewpoint for arbitrary homogeneous dielectric media. The proof of equivalence in summation via Casimir-Polder - van der Waals type potentials and Casimir energy in the lowest $(\varepsilon-1)^{2}$ order is the subject of section 3. This equivalence made it possible to calculate non-dispersive contribution to Casimir energy of a dilute dielectric ball by direct summation of Casimir-Polder potential. When the number of atoms constituting dilute ball is assumed constant during the ball expansion, the Casimir surface force is attractive and includes volume and surface terms in the order $(\varepsilon-1)^{2}$.

\section{Appendix}

For the benefit of the reader it is helpful to give here an overview of different approaches which have been used to derive the finite when $\lambda \rightarrow 0$ term $E_{f}=23 /(1536 \pi a)$ in the energy expression (45).

First attempts to calculate Casimir energy of a dielectric ball gave a lot of different answers, as it is described in [13]. The mathematical reason for some of these differences was found in the work [14, where by use of Debye expansion for Bessel functions and $\zeta$-function the correct limits on $E_{f}$ were established. Later in the article [15] the term $E_{f}$ was calculated numerically with high accuracy, which made it possible to establish equivalence of Casimir effect and retarded van der Waals (or Casimir-Polder) energy for the case of non-dispersive dielectric ball. 
Mutual retarded van der Waals energy for molecules inside the compact sphere (ball) was calculated by K.Milton and Y.Ng [16 and its finite part after regularization in terms of gamma functions was first given by $E_{f}$ in the work [16].

In the article 15] Casimir energy of a dilute dielectric ball was studied via the formula which has the following form for nondispersive case:

$$
E_{C}=-\frac{(\varepsilon-1)^{2}}{8 \pi a} \sum_{l=1}^{\infty}(2 l+1) \int_{0}^{\infty} d x x \frac{d}{d x} F_{l}(x),
$$

where

$$
F_{l}(x)=-\frac{1}{4}\left(\frac{d}{d x}\left(e_{l} s_{l}\right)\right)^{2}-x^{2}\left(s^{\prime 2}-s s^{\prime \prime}\right)\left(e^{\prime 2}-e e^{\prime \prime}\right),
$$

and Riccati-Bessel functions are assumed to depend on argument $x$.

The formula (46) can be studied using formula (18) as well. We put $k=1, p=1-m$, where we are interested in the limit $m \rightarrow 0$. For $E_{C}$ we find

$$
E_{C}=\lim _{m \rightarrow 0} \frac{(\varepsilon-1)^{2}}{\pi a}\left(\frac{23}{1536}+O\left(\frac{1}{m}\right)\right) .
$$

Recently the formula (46) has been studied in the mode summation method [17], there divergent terms have been analysed and the finite result $E_{f}$ has also been derived by making use of addition theorem for Bessel functions.

Another approach based on quantum mechanical perturbation theory was suggested by G.Barton in the work [13]. The Casimir energy was obtained there in the form:

$$
\begin{aligned}
E=-(\varepsilon-1) & \frac{3}{2 \pi^{2}} \frac{V}{\lambda^{4}}+ \\
& +(\varepsilon-1)^{2}\left(-\frac{3}{128 \pi^{2}} \frac{V}{\lambda^{4}}+\frac{7}{360 \pi^{3}} \frac{S}{\lambda^{3}}-\frac{1}{20 \pi^{2}} \frac{1}{\lambda}+\frac{23}{1536 \pi} \frac{1}{a}\right) .
\end{aligned}
$$

Here $V$ is the volume and $S$ the surface area, $1 / \lambda$ is an exponential cutoff on wavenumbers. In this approach the contact terms haven't been subtracted, this is why the term proportional to $\varepsilon-1$ is present in (49). We have already discussed this formula in section 4 , its structure is similar to (45). The cutoff independent term is essentially the same in all approaches.

The approach based on quantum statistical mechanics was developed in [18. This work led to similar results. 
The theory of QED in a dielectric background was studied in [3] by path integral and $\zeta$-function methods. $\zeta$-function can be written as in [3]:

$$
\zeta(s)=\frac{\sin \pi s}{\pi} \sum_{l=1}^{\infty}(2 l+1) \int_{0}^{\infty} d \omega \omega^{-2 s} \frac{\partial}{\partial \omega} \ln \left(\Delta_{l} \tilde{\Delta}_{l}\right) .
$$

For a dilute ball it is possible to expand the logarithm in powers of $\varepsilon-1$, here we consider only the order $(\varepsilon-1)^{2}$ of this expansion:

$$
\zeta(s)=\frac{(\varepsilon-1)^{2}}{4} \frac{\sin \pi s}{\pi} \sum_{l=1}^{\infty}(2 l+1) \int_{0}^{\infty} d \omega \omega^{-2 s} \frac{\partial}{\partial \omega} F_{l}(\omega a),
$$

where $F_{l}(x)$ is defined in (47). Detailed derivation of the finite part of $\zeta$ function deserves a single article, here for completeness we only present the result for its finite part $\zeta_{f}$ :

$$
\zeta_{f}(s)=-(\varepsilon-1)^{2} \frac{\sin (\pi s)}{\pi s} a^{2 s} 2^{4 s-7} \frac{\left(s^{2}-3 s+4\right) \Gamma(-2 s+2)}{(s-1)},
$$

with no poles in this expression for $s<1$. From this expression we find

$$
E_{f}=\frac{\zeta_{f}(-1 / 2)}{2}=\frac{23}{1536} \frac{(\varepsilon-1)^{2}}{\pi a},
$$

we only note that even $\zeta$-function method meets severe problems with analytic continuation and regularization in the case of a dilute dielectric ball.

Most divergences and problems met so far in different approaches are related to short distance behaviour. In this article it is shown explicitly that such problems arise when parameter $\lambda \rightarrow 0$. In dielectric media in the real world $\lambda \neq 0$, this is why the main problem which still remains in other approaches is not to remove regularization, but to identify parameters of regularization in final expressions with physical quantities.

\section{References}

[1] J.Schwinger, Proc.Natl.Acad.Sci. USA 90 (1993), 958, 2105, 4505, 7285; 91 (1994), 6473.

[2] B.P.Barber, R.A.Hiller, R.Löfstedt, S.J.Putterman and K.Weniger, Phys.Rep.C 281 (1997), 65.

[3] M.Bordag, K.Kirsten and D.Vassilevich, hep-th/9811015, Phys.Rev.D 59 (1999), 085011. 
[4] E.M.Lifshitz and L.P.Pitaevskii, "Statistical Physics", Part 2 (Course of Theoretical Physics, vol. IX).

[5] K.A.Milton, L.L.DeRaad,Jr. and J.Schwinger, Ann.Phys.(N.Y.) 115 (1978), 1,388.

[6] I.Brevik, M.Lygren and V.N.Marachevsky, Ann.Phys.(N.Y.) 267 (1998), 134.

[7] K.A.Milton and Y.J.Ng, hep-th/9607186, Phys.Rev.E 55 (1997), 4207.

[8] J.D.Jackson, "Classical Electrodynamics", 2nd ed., John Wiley, New York, 1975.

[9] M.Abramowitz and I.Stegun, "Handbook of Mathematical Functions", 9th ed., Dover, New York, 1972.

[10] I.Klich, hep-th/9908101, Phys.Rev.D 61 (2000), 025004.

[11] H.B.G. Casimir and D.Polder , Phys.Rev. 73 (1948), 360.

[12] V.B.Berestetskii, E.M.Lifshitz and L.P.Pitaevskii , "Quantum Electrodynamics" (Course of Theoretical Physics, vol. IV ).

[13] G.Barton, J.Phys. A 32 (1999), 525.

[14] I.Brevik and V.Marachevsky, Trondheim preprint "Casimir surface force on a dilute dielectric ball" (1998), hep-th/9901086, Phys.Rev.D 60 (1999), 085006.

[15] I.Brevik, V.N.Marachevsky and K.A.Milton, hep-th/9810065,

Phys. Rev. Lett.82 (1999), 3948.

[16] K.A.Milton and Y.J.Ng, Phys.Rev.E 57 (1998), 5504.

[17] G.Lambiase, G.Scarpetta and V.V.Nesterenko, "Exact value of the vacuum electromagnetic energy of a dilute dielectric ball in the mode summation method", hep-th/9912176.

[18] J.S.Hoye and I.Brevik, "The Casimir problem of spherical dielectrics: a solution in terms of quantum statistical mechanics", quant-ph/9903086. 